\title{
THE ROLE OF GRAINS IN MOLECULAR CHEMICAL EVOLUTION*
}

J. M. GREENBERG

University of Leiden, Laboratory Astrophysics, Leiden, The Netherlands

(Received 27 June, 1986)

\begin{abstract}
The observations of dust gas in diffuse and molecular clouds are shown to reflect not only their current state but their past history. The interpretation of infrared spectra of dust in molecular clouds using appropriate core-mantle grains shows that: (1) the kinds and amounts of ices, (2) the relative proportion of such important interstellar molecules as $\mathrm{H}_{2} \mathrm{O}$ and $\mathrm{CO}$, (3) the evidence for the less abundant solid species $\mathrm{X}-\mathrm{C} \equiv \mathrm{N}, \mathrm{COS}, \mathrm{H}_{2} \mathrm{~S}$, and (4) the thermal history of the dust may all be demonstrated quantitatively from laboratory analog studies of ultraviolet photoprocessing of relevant ices and from theoretical studies of gas-dust interactions. In diffuse clouds the dust is shown to consist predominantly of refractory organic compounds which originate as residues of the photoprocessing of volatile ices in molecular clouds and which undergo further physical and chemical evolution in the diffuse clouds.
\end{abstract}

\section{Introduction}

Chemical evolution in the space between the stars follows a complex series of steps involving ions, electrons, atoms, and molecules in the gas and on and in the small solid interstellar dust grains. The role of grains is a very important one in determining the relative amounts of some of the most important molecules. For example, although the $\mathrm{H}_{2} \mathrm{O}$ on grains is observed to be more abundant than $\mathrm{CO}$ (van de Bult et al., 1985; Whittet et al., 1985; Lacy et al., 1984) just the opposite is true in the gas (Mann and Williams, 1980).

The principal processes in the overall chemical evolution scheme involving the grains are accretion and ultraviolet photoprocessing of grain mantles. The study of these processes has been made possible by the creation of laboratory conditions which simulate those relevant to the evolution of grains in interstellar space. The key experimental components are the low temperature, low pressure, and the ultraviolet. These have been amply discussed in Hagen et al. (1979) and Van IJzendoorn (1985). We have studied infrared spectra of various molecular mixture both qualitatively and quantitatively, finding for some solid mixtures the values of the complex index of refraction $m=m^{\prime}-i m^{\prime \prime}$ as a function of temperature. We have produced stored radicals and studed their diffusion and, in some cases, the explosive reactions caused by sudden heating (d'Hendecourt et al., 1982). All of these results have been applied, as seen in the following selected brief summaries, to simulating grains conditions which may be either directly or indirectly observed by astronomical means.

\footnotetext{
* Review paper presented at a Workshop on 'The Role of Dust in Dense Regions of Interstellar Matter', held at Georgenthal, G.D.R., in March 1986.
}

Astrophysics and Space Science 128 (1986) 17-31.

(C) 1986 by D. Reidel Publishing Company 
A basic fact which emerges is that the evolution of grains is a cyclic one in which the grains pass sequentially from shielded molecular cloud conditions to exposed diffuse cloud conditions many times during their full lifetime (Greenberg, 1982a, b, 1986).

\section{The Ice Band}

The $3.07 \mu \mathrm{m} \mathrm{OH}$ stretch gets special attention because it is by far the dominant infrared feature of grain mantles. It has proven to be not only a probe of the chemical but also the physical evolution of dust.

Two examples of ice absorption observations (Whittet et al., 1983) are shown in Figure 1 and compared with a reference absorption of crystalline $\mathrm{H}_{2} \mathrm{O}$. We note that the width and peak absorption of the grains in the line-of-sight to HL Tau is closer to that of crystalline ice than that of Elias 16. The values of $m^{\prime}$ and $m^{\prime \prime}$ as applied to the calculation of the absorption cross sections of various shapes of particles show that the absorption structures shown in Figure 1 are best matched by long particles whether cylindrical or spheroidal (van de Bult et al., 1985; Greenberg et al., 1983) and that the shape of the particle is almost as important as the choice of the ice mixture in determining the degree of matching. One may deduce from the absorption strength, width and peak positions of the ice band in Elias 16 and HL Tau that the fractional amount of $\mathrm{H}_{2} \mathrm{O}$ in the grain mantles is greater than $50 \%$ and, in fact is probably as much

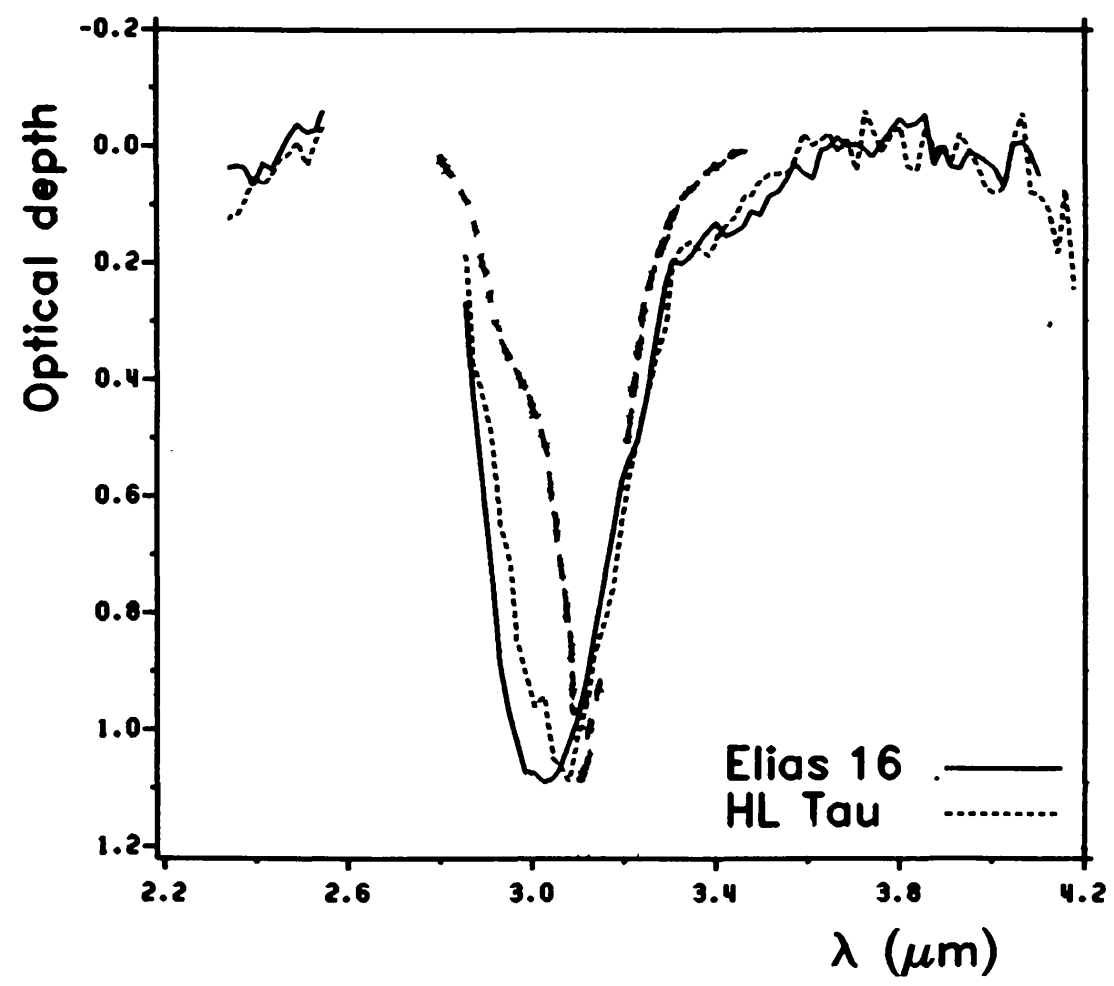

Fig. 1. Comparison of the observed spectra of HLK Tau and Elias 16 normalized to the same optical depth and with straight lines connecting the points. Dashed curve is absorption by crystalline ice (Bertie et al., 1986). 

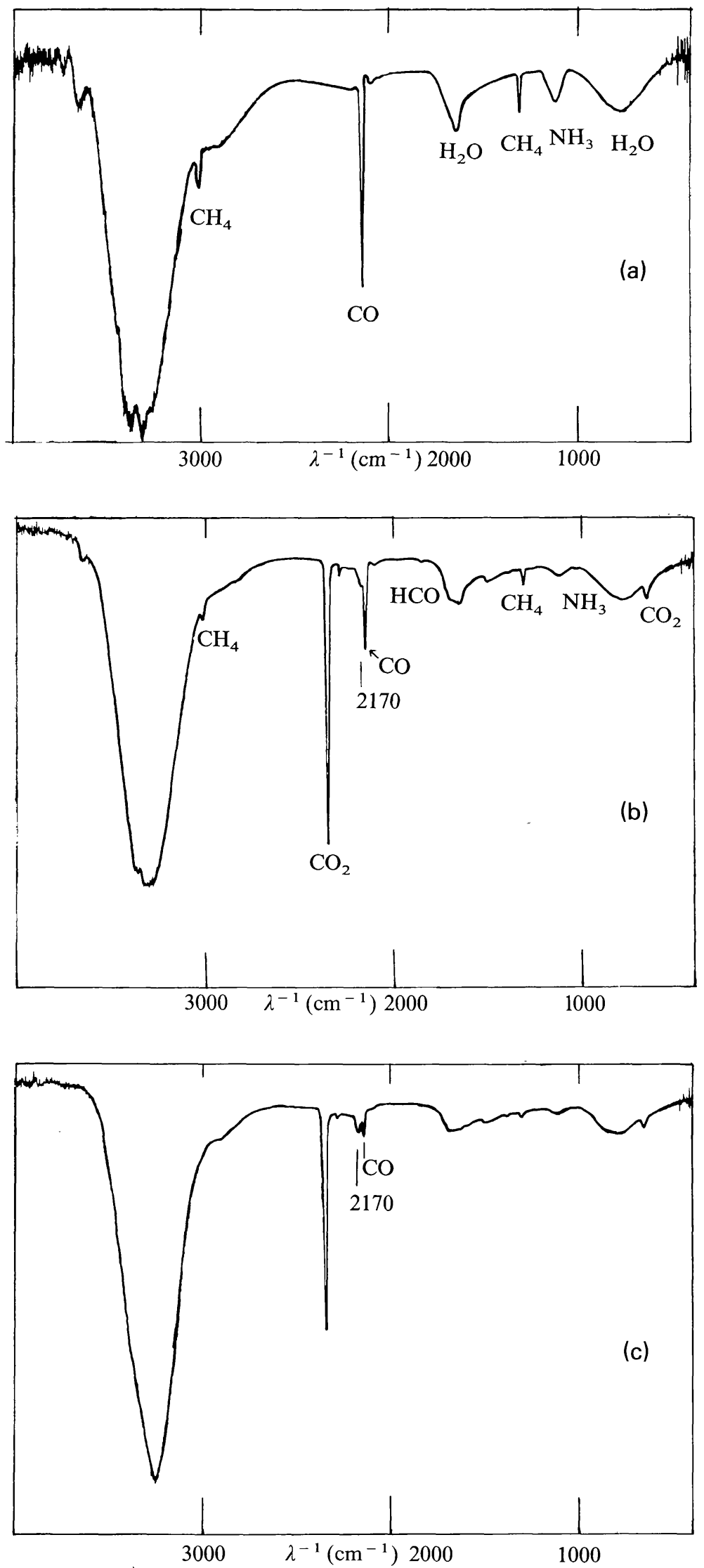

Fig. 2. Spectra of a water rich mixture $\left(\mathrm{H}_{2} \mathrm{O}: \mathrm{CO}: \mathrm{NH}_{3}: \mathrm{CH}_{4}=6: 2: 1: 1\right)$. (a) Non-irradiated $10 \mathrm{~K}$; (b) photolyzed; (c) photolyzed and warmed up to $95 \mathrm{~K}$. 
as $60-70 \%$. Furthermore, while the Elias 16 ice band is characteristic of mantles formed and kept at the very low temperature of $\lesssim 15 \mathrm{~K}$, the HL Tau ice band clearly shows annealing by warmup to at least $50 \mathrm{~K}$. Since Elias 16 is behind the Taurus molecular cloud it samples the general average of dust mantles which have formed there. On the other hand HL Tau is known to have a high degree of local activity probably in the form of an accretion disk (Cohen, 1983) so that the dust in its vicinity is likely to have undergone a significant amount of heating.

\section{Photoprocessing of Grain Mantles}

The ultraviolet irradiation of a water rich ice mixture $\left(\mathrm{H}_{2} \mathrm{O}: \mathrm{CO}: \mathrm{NH}_{3}: \mathrm{CH}_{4}=\right.$ $6: 2: 1: 1$ ) is shown in Figure 2 (d'Hendecourt et al., 1986). The initial stage is that of a deposit without irradiation which shows infrared absorption features belonging to the initial molecular constituents. Next shown is the result of $5 \mathrm{hr}$ irradiation corresponding to about a million years in a molecular cloud. Many new features are seen among which the obviously strong one of $\mathrm{CO}_{2}$. The weak feature of $\mathrm{HCO}$ indicates about 0.1 to $1 \%$ radical concentration. The short wavelength wing at $4.62 \mu \mathrm{m}\left(2170 \mathrm{~cm}^{-1}\right)$ appearing on the $\mathrm{CO}$ absorption at $4.67 \mu \mathrm{m}$ has particular significance because it persists at relatively high temperatures while $\mathrm{CO}$ is readily evaporated as shown in the spectrum after the mixture has been warmed to $95 \mathrm{~K}$. This effect has also been observed in W33A (see Figure 3) where the $4.62 \mu \mathrm{m}$ absorption has been identified as belonging to a

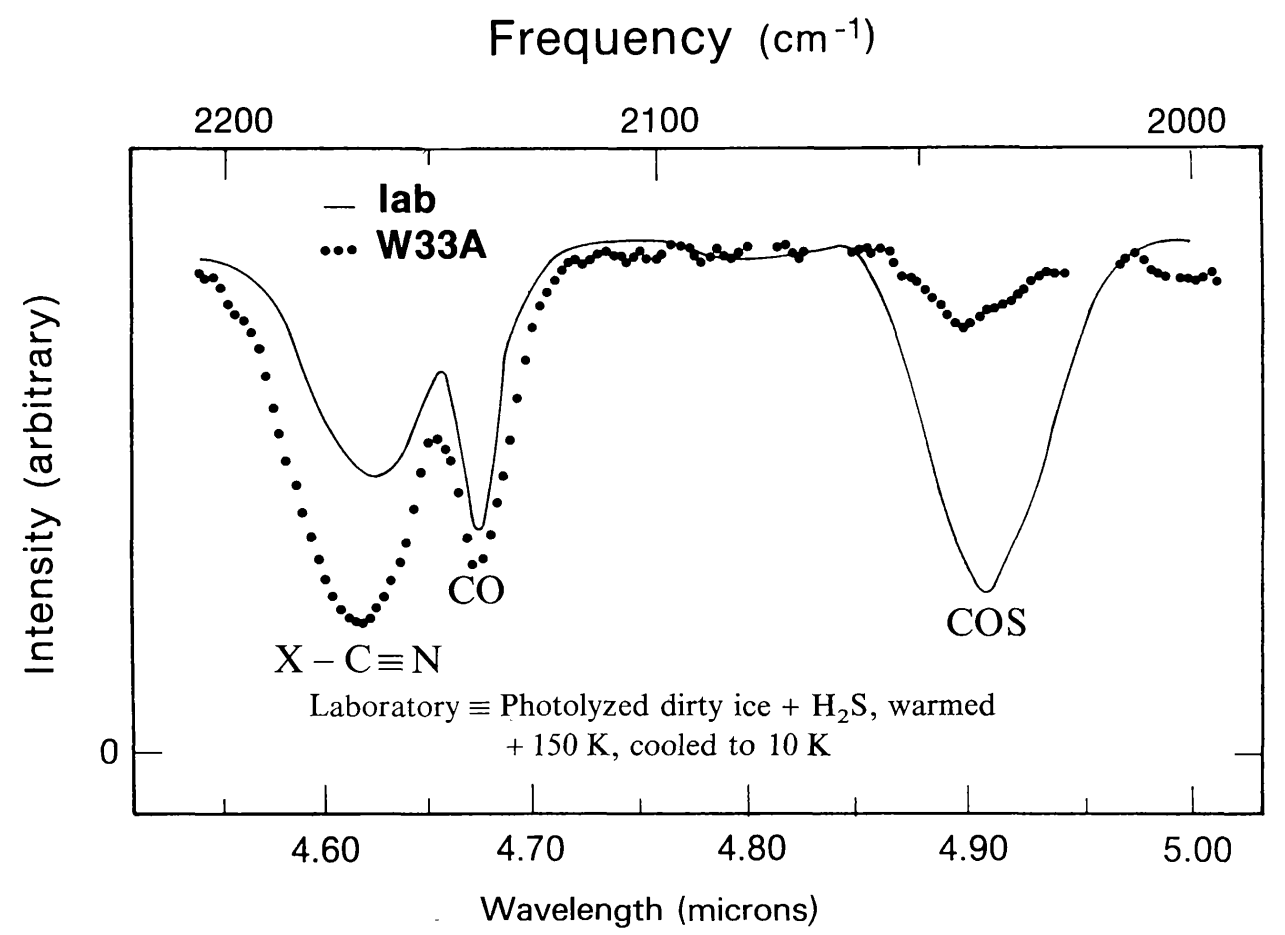

Fig. 3. Laboratory spectrum of a frozen mixture of simple molecules, including $\mathrm{H}_{2} \mathrm{~S}, \mathrm{CO}$, and $\mathrm{NH}_{3}$, which has been subjected to UV-irradiation at $10 \mathrm{~K}$ and then warmed to $85 \mathrm{~K}$. The spectrum of W33A is shown for comparison with its baseline flattened. 


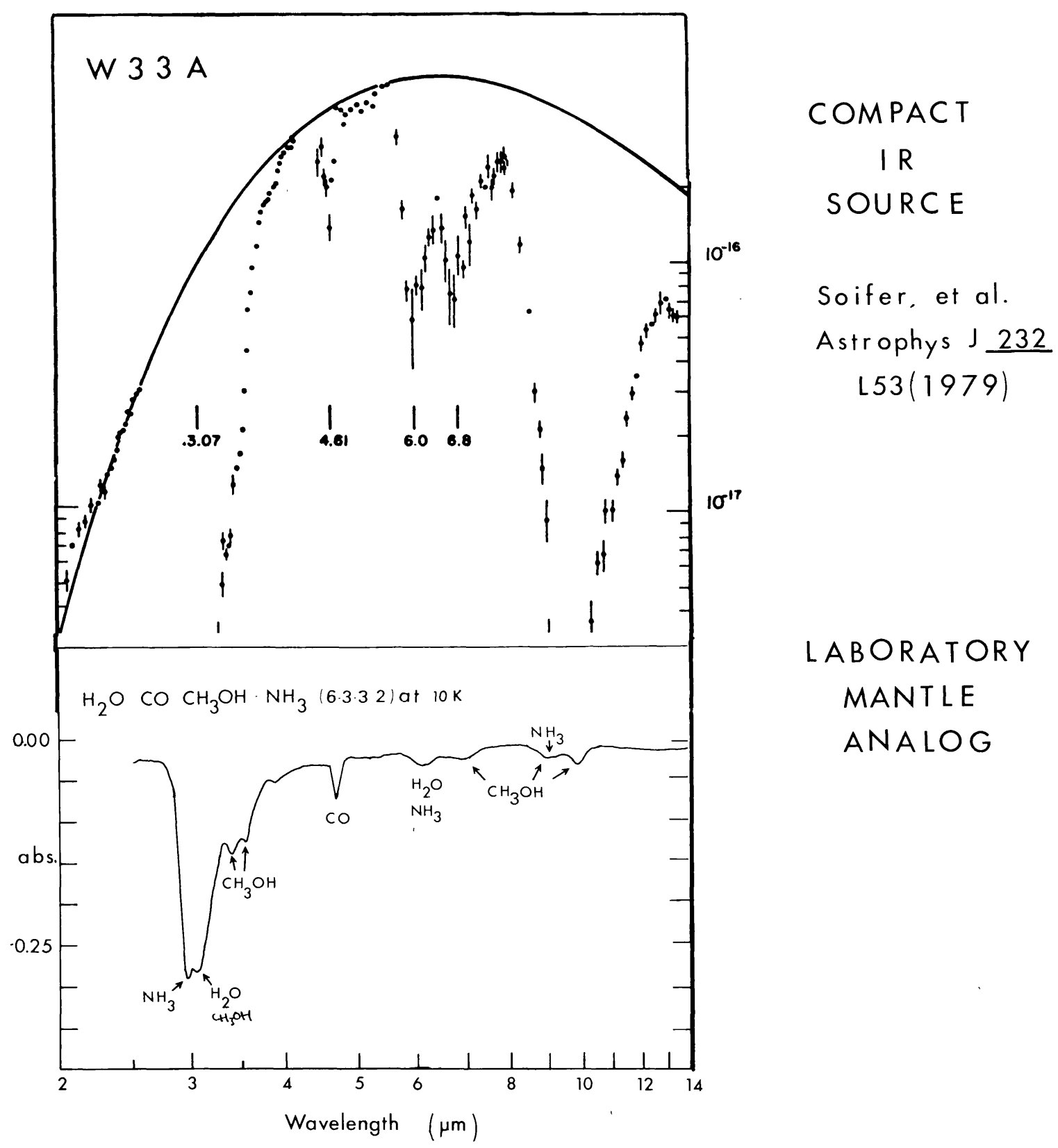

Fig. 4. Spectrum of a compact source W33A showing several features in addition to the strong $\mathrm{H}_{2} \mathrm{O}$ and silicate absorptions at 3.07 and $9.5 \mu \mathrm{m}$. For comparison is shown a spectrum of a laboratory mantle analog mixture.

molecule (as yet unidentified) containing the cyanogen group $-\mathrm{C} \equiv \mathrm{N}$ (Lacy et al., 1984). The deeply dust imbedded object W33A has also provided us with evidence of photoprocessing of grain mantles containing $\mathrm{H}_{2} \mathrm{~S}$ (seen at $3.9 \mu \mathrm{m}$ ) and producing $\mathrm{COS}$ (carbonyl sulfide). We note how the amount of $\mathrm{CO}$ relative to $\mathrm{H}_{2} \mathrm{O}$ in $\mathrm{W} 33 \mathrm{~A}$ is indeed small when we see the full spectrum shown in Figure 4 (Soifer et al., 1979). 


\section{Grain Explosions}

Irradiation at low temperatures leads to the production and storage of free radicals in the ices. This highly reactive mixture, when slowly warmed produces chemiluminescence and thermoluminescence which have been studied in some detail in various mixtures (Van IJzendoorn, 1985). When the grains are maintained at temperatures $T_{d} \lesssim 15 \mathrm{~K}$ essentially all the radicals remain for times $\sim 10^{8} \mathrm{yr}$. However, if the dust temperature is suddenly raised to about $27 \mathrm{~K}$, laboratory results show that enough radicals diffuse and recombine (releasing stored energy) to lead to a chain reaction which explodes the mixture. In an interstellar cloud such temperatures may be created either by grain-grain collisions at velocities $>40 \mathrm{~m} \mathrm{~s}^{-1}$ (Greenberg, 1979; d'Hendecourt et al., 1983) in a turbulent medium or by bombardment by cosmic-ray iron ions (Léger et al., 1985). This explosive desorption of molecules from the dust mantles appears to be adequate to maintain the gas phase species against total depletion onto the grains.

\section{Organic Refractory Grain Mantles}

Cosmic abundance arguments show that silicates alone are inadequate to provide the amount and wavelength dependence of extinction (Greenberg and Hong, 1975). An appropriate combination of silicates with graphite may satisfy the extinction requirements (Mathis et al., 1979), but the amount of polarization and the relation between linear and circular polarization seem to demand that the major portion of the extinction be produced by the dialectric grians (Greenberg, 1986; Aannestad and Greenberg, 1983). Although this implies that the silicate grains which produce the $9.5 \mu \mathrm{m}$ absorption

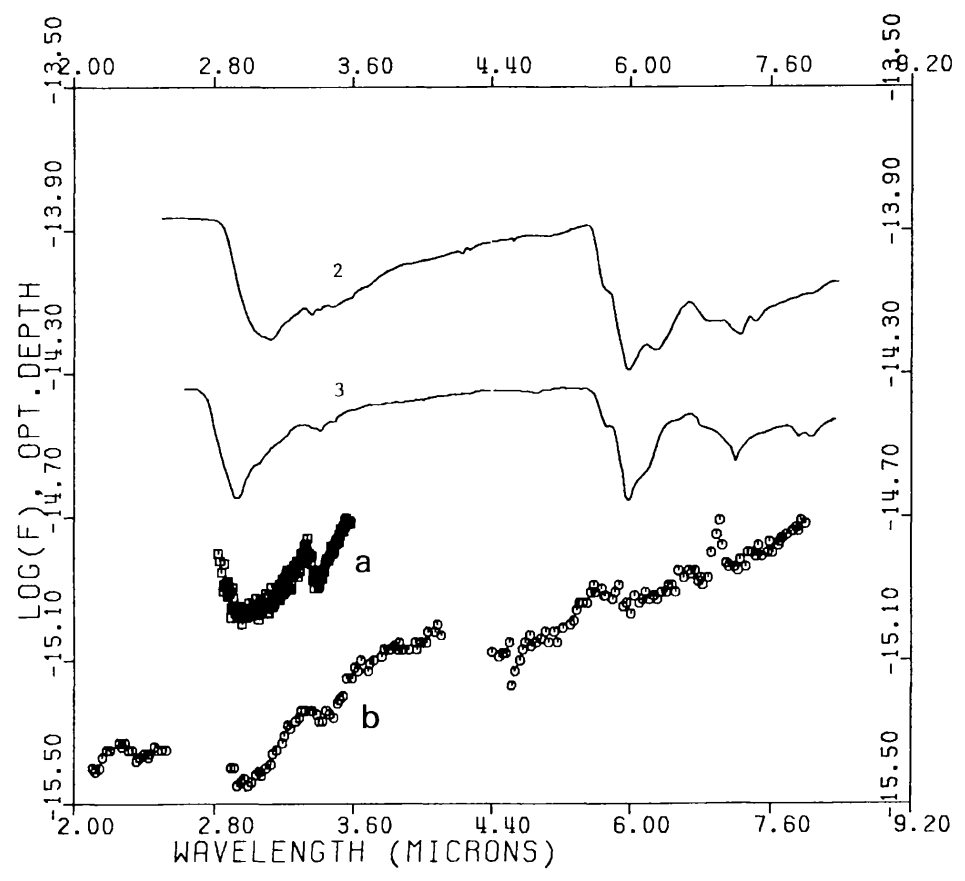

Fig. 5a. 


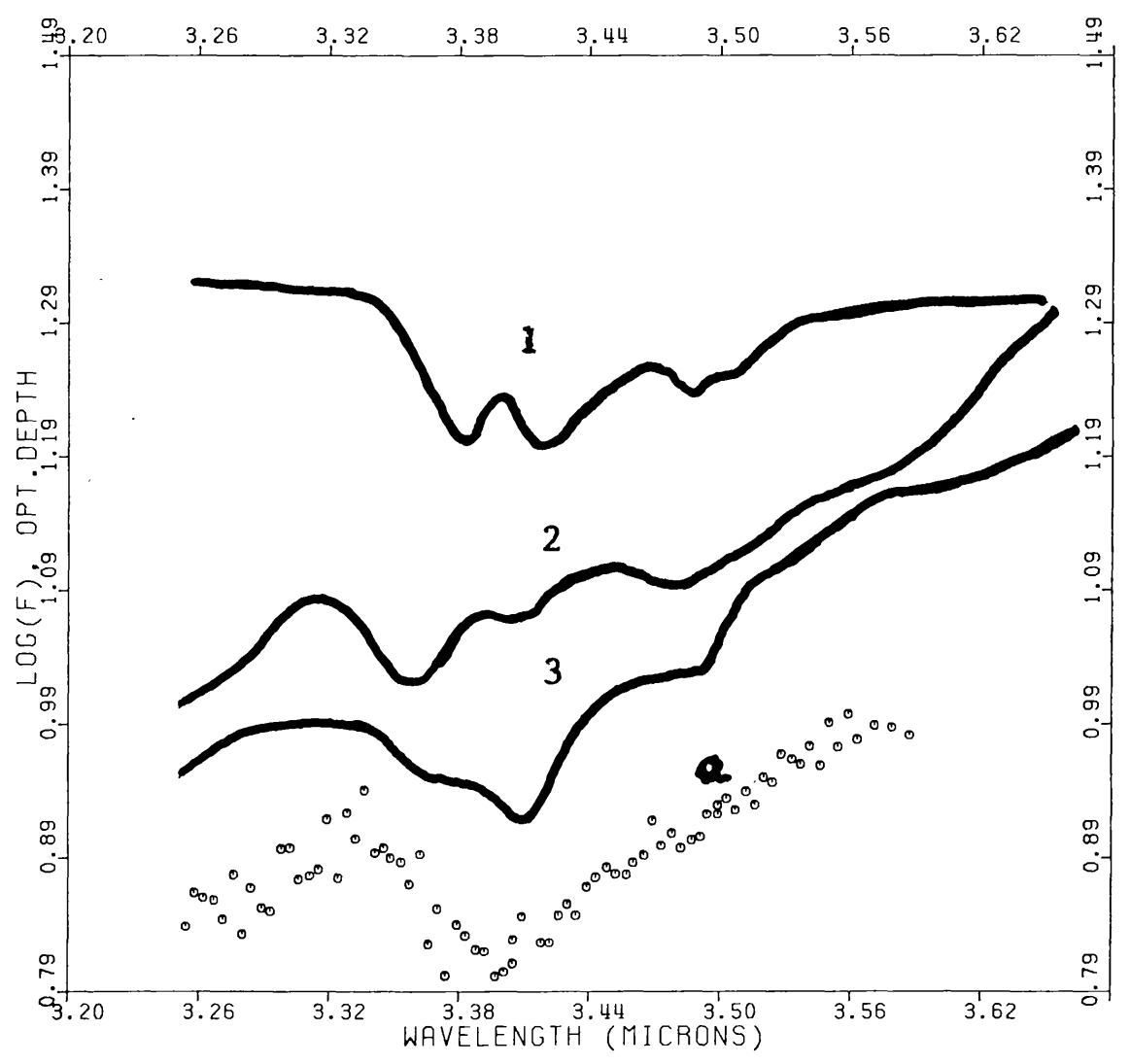

Fig. 5b.

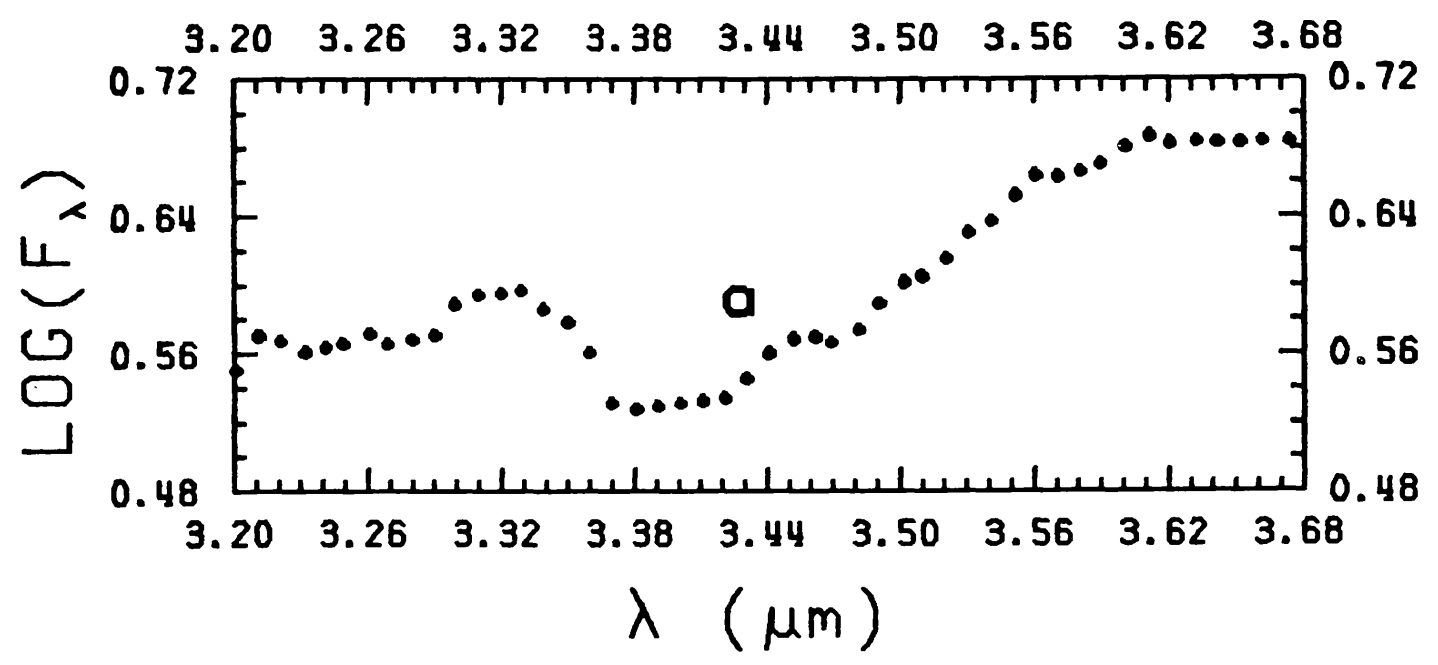

Fig. 5c.

Fig. 5a-c. Infrared absorption spectra of several organic refractory residues and the galactic center sources IRS7 and Sgr AW. (1) Residue of $\mathrm{CH}_{4}$, (2) residue of $\mathrm{CO}: \mathrm{H}_{2} \mathrm{O}: \mathrm{CH}_{4}: \mathrm{NH}_{3}=2: 2: 1: 2$, (3) residue of $\mathrm{CO}: \mathrm{H}_{2} \mathrm{O}: \mathrm{NH}_{3}=5: 5: 1$, (a) IRS7, (b) Sgr AW. Separate curve for $3.4 \mu \mathrm{m}$ feature IRS7 is fully smoothed and shows a peak absorption somewhere between that of residues 2 and 3 . 
must be coated with mantles which contain the organics $\mathrm{O}, \mathrm{C}$, and $\mathrm{N}$, there is no evidence that the normally abundant $\mathrm{H}_{2} \mathrm{O}$ of molecular cloud grain mantles exist in adequate amount in the diffuse cloud medium. Thus, another form of grain mantle which contains $\mathrm{O}, \mathrm{C}$, and $\mathrm{N}$ must exist. This form is produced as the result of ultraviolet photoprocessing of ices leading to organic refractory molecular mixtures (Greenberg et al., 1972; Hagen et al., 1979). The direct evidence for organic residues in space was finally exhibited in the observation of the $3.4 \mu \mathrm{m}$ feature toward objects in the galactic center as in Sgr AW (Willner et al., 1979). This feature has been further observed with much better resolution in IRS 7 (Butchart et al., 1986). Laboratory residues whether produced by ultraviolet (see Figure 5) or proton bombardment (Moore and Donn, 1982; Strazzulla et al., 1984) have not produced a precise match to the observed $3.4 \mu \mathrm{m}$ structure although the UV irradiated residues appear closest to the observations. On the other hand the measured rate of formation by ultraviolet photoprocessing in molecular clouds has been shown to provide an absorption strength which is adequate to account for the mantles on the dust grains in the diffuse cloud medium along the distance to the galactic center (Schutte and Greenberg, 1986). The variety of absorptions in the $3 \mu \mathrm{m}$ region observed towards the galactic center (Figure 6) shows that some, even though not much, $\mathrm{H}_{2} \mathrm{O}$ must exist along that line-of-sight implying the presence or a bit of molecular

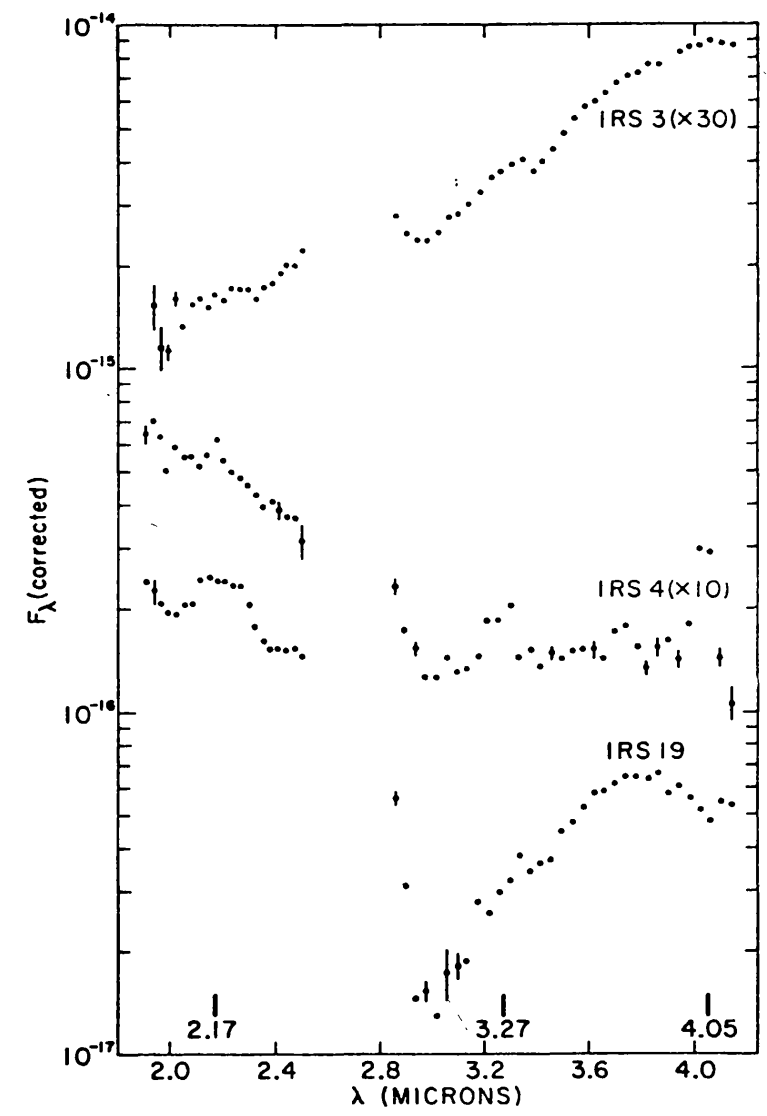

Fig. 6. Spectra of galactic center sources corrected for interstellar extinction. Note the variety of absorptions peaked at about $3 \mu \mathrm{m}$ evidently not due to normal $\mathrm{H}_{2} \mathrm{O}$. 
cloud dust extinction (Schutte and Greenberg, 1986). In any case, the presence of organic refractory mantles is clealry proven so that, in general, the grains in molecular clouds must consist of silicate cores with layered mantles, the inner portions being the residues which have been produced in molecular clouds and which have undergone long term irradiation in the diffuse cloud medium. The outer portion in molecular clouds is an irradiated icy mixture and in diffuse clouds is the latest formed residue.

\section{Grain Mantle and Gas Evolution in Molecular Clouds}

The interplay between the atoms and molecules in the gas and in the grains consists of essentially five basic steps: (1) ion-molecule reactions in the gas, (2) atom and molecule sticking on grains, (3) grain surface reactions, (4) ultraviolet irradiation and production of stored radicals, (5) grain explosions replenishing the gas (d'Hendecourt et al., 1985). The state of the grains and gas is a time-dependent one even if one maintains constant cloud conditions. The time-dependence is, of course, further modified if we allow the cloud itself to evolve or change. This remains to be considered in the future. For the moment I present a small sample of the results applicable to a rather dense cloud of density $n_{\mathrm{H}}=2 \times 10^{4} \mathrm{~cm}^{-3}$ and a mean ultraviolet attenuation relative to the diffuse cloud medium of $\sim 3 \times 10^{-4}$. This leads to an ultraviolet irradiation time for the production of $1 \%$ radicals of less than $10^{5} \mathrm{yr}$ which is adequate to provide explosive mixtures between grain-grain collision times.

In Figures 7(a), (b), and (c) the time-dependence of the mantle components show several very important consequences.

(1) At early times the grain mantles are dominated by $\mathrm{H}_{2} \mathrm{O}, \mathrm{CH}_{4}$, and $\mathrm{NH}_{3}$ in relative amounts more or less as given by Van de Hulst (1949).

(2) At intermediate times $\left(10^{5}-10^{7} \mathrm{yr}\right)$ the mantle remains dominated by $\mathrm{H}_{2} \mathrm{O}$ and would ultimately contain a very large $\mathrm{CO}$ concentration if we neglect the photoprocessing which leads to a large concentration of $\mathrm{CO}_{2}$ (an effect neglected in this calculation).

(3) At longer times, $\mathrm{H}_{2} \mathrm{O}+\mathrm{CO}_{2}$ remain the dominant constituents with no significant amount of $\mathrm{CH}_{4}$ or $\mathrm{NH}_{3}$.

The mantle composition is at all times very different from the gas composition and notably this is exhibited by the fact that the $\mathrm{H}_{2} \mathrm{O}$ in the dust is never less than 10 times that in the gas so that water ice is not accreted on grains it is created on grains. This is why the $\mathrm{H}_{2} \mathrm{O}$ in grain mantles is always observed to be larger than the $\mathrm{CO}$ even though, in the gas, the $\mathrm{CO}$ is by far the dominant molecular constituent. The theoretically predicted drop in the $\mathrm{H}_{2} \mathrm{O}$ mantle fraction in dense clouds for $t>10^{7} \mathrm{yr}$ is probably prevented from generally occurring because such clouds are not likely to be stable over times as long as this either as a consequence of cloud-cloud collisions or cloud contraction. Thus a canonically stable value of the fraction of $\mathrm{H}_{2} \mathrm{O}$ in molecular cloud dust outer mantles is probably about $60-70 \%$ as already noted for Elias 16 and even for the B.N. ice mantles where they exist (Greenberg, 1982b).

The solid CO which has been observed (Whittet et al., 1985) in Elias 16 representing 


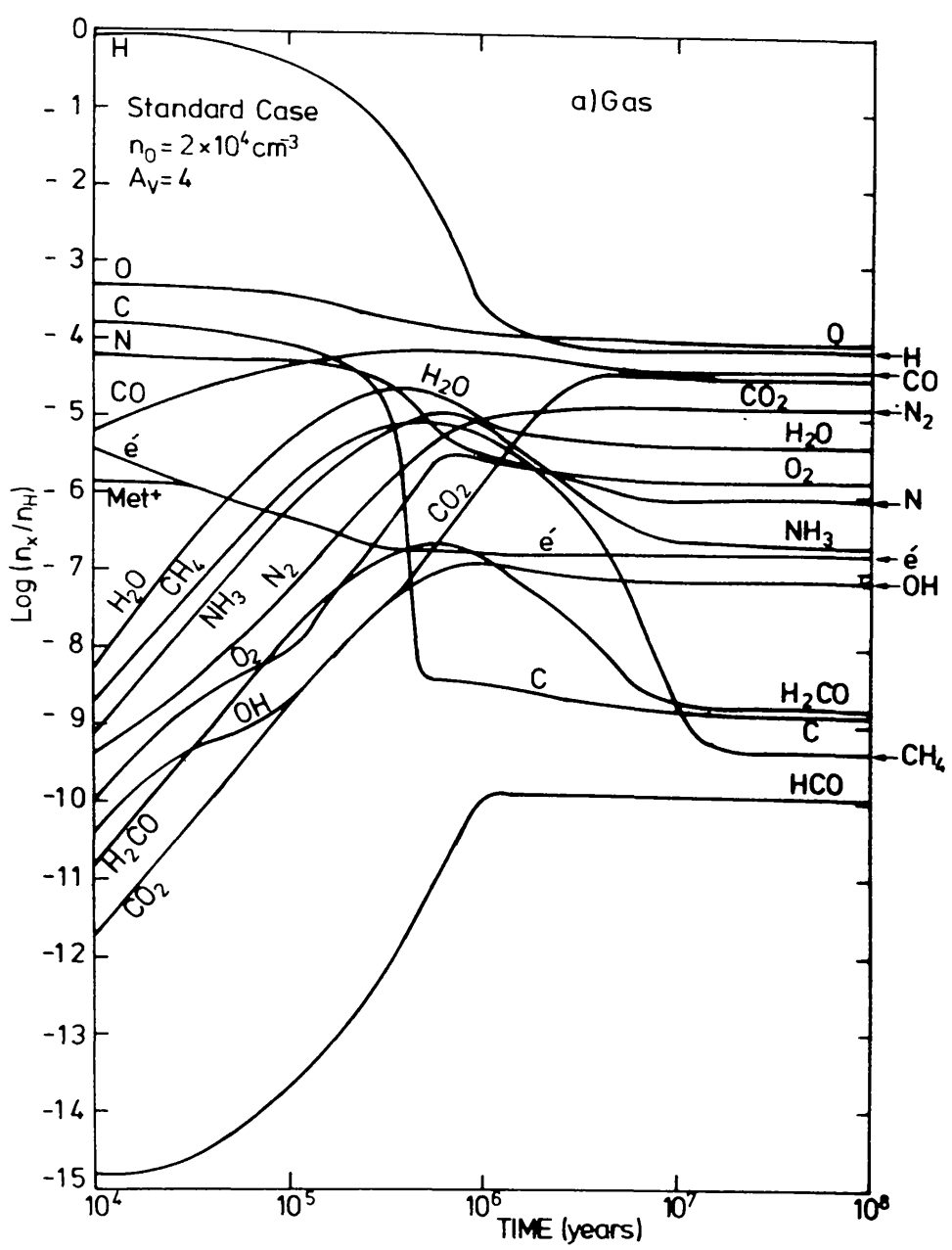

Fig. 7a. Time-evolution of the abundances of the molecular components of the gas in a cloud with density $n_{\mathrm{H}}=2 \times 10^{4} \mathrm{~cm}^{-3}$ and extinction $A_{V}=4 \mathrm{mag}$.

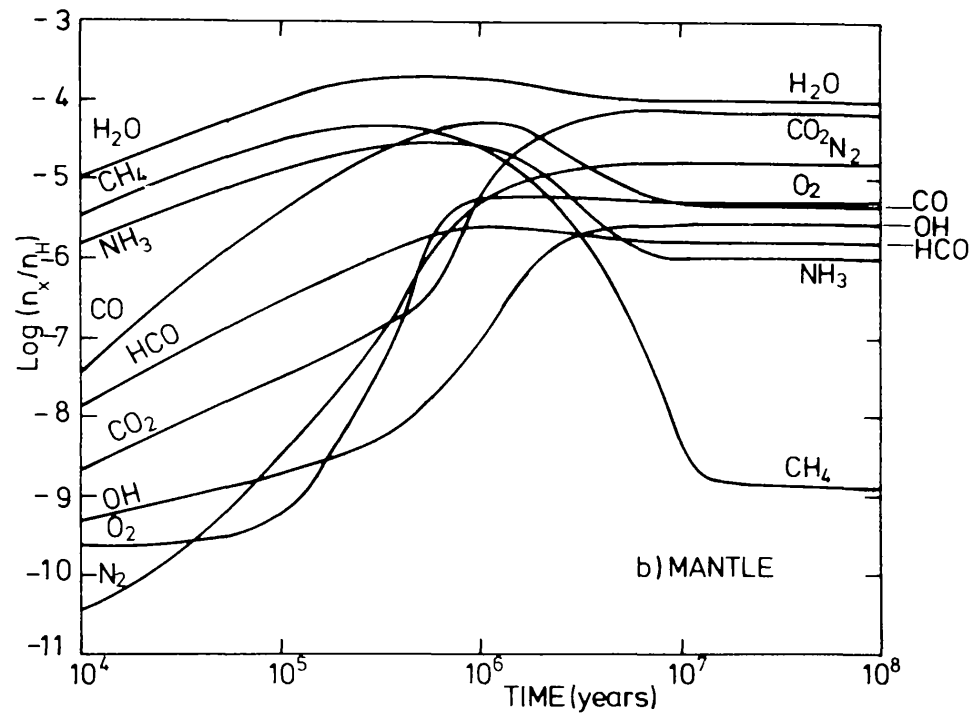

Fig. 7b. Same as Figure 7(a) for the grain mantle. 


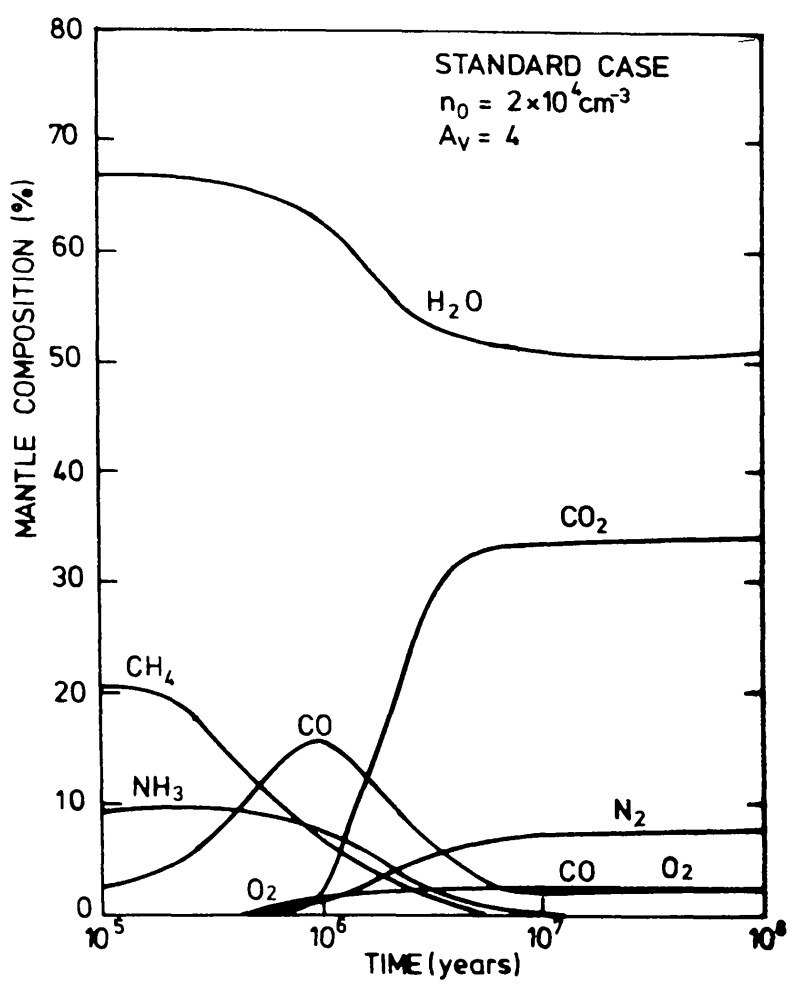

Fig. 7c. Relative fractions of the major volatile components of the grain mantles in a cloud with $n_{\mathrm{H}}=2 \times 10^{4} \mathrm{~cm}^{-3}$ and extinction $A_{V}=4 \mathrm{mag}$.

normal molecular cloud dust is less abundant than the solid $\mathrm{H}_{2} \mathrm{O}$ even though the $\mathrm{CO} / \mathrm{H}_{2} \mathrm{O}$ in the gas is $\gg 1$. It was shown by van de Bult et al. (1985) that the fraction of $\mathrm{H}_{2} \mathrm{O}$ in the outer icy grain mantles is about $60 \%$ and is unheated. The relative amounts of $\mathrm{H}_{2} \mathrm{O}$ and $\mathrm{CO}$ are given by the ratio $n_{\mathrm{H}_{2} \mathrm{O}} / n_{\mathrm{CO}}=\left(\sigma_{\mathrm{CO}} / \sigma_{\mathrm{H}_{2} \mathrm{O}}\right)\left(\tau_{\mathrm{H}_{2} \mathrm{O}} / \tau_{\mathrm{CO}}\right)$ where the cross section per $\mathrm{CO}$ molecule is about $\sigma_{\mathrm{CO}} \simeq 1.3 \times 10^{-18} \mathrm{~cm}^{2}$ (Lacy et al., 1984) and the cross section per $\mathrm{H}_{2} \mathrm{O}$ molecule is $\left.\sigma_{\mathrm{H}_{2} \mathrm{O}}^{f} \simeq((f-0.15) / 0.85)\right) \sigma_{\mathrm{H}_{2} \mathrm{O}}^{\text {pure }}$ where $f=\mathrm{H}_{2} \mathrm{O}$ fraction in the grains and 'pure' stands for pure amorphous ice. The reduction factor $(f-0.15) / 0.85$ results from the statistical proportion of polymeric ice (Greenberg et al., 1983). We derive $\sigma_{\mathrm{H}_{2} \mathrm{O}}^{\text {pure }}=0.6 \times 10^{-18} \mathrm{~cm}^{2}$ by inserting the measured absorptive part of the index of refraction, $m^{\prime \prime}(3.07)=0.477$, in the relation, $4 \pi m^{\prime \prime} / \lambda=s\left(M m_{\mathbf{H}}\right)^{-1} \sigma_{\mathrm{H}_{2} \mathrm{O}}^{\text {pure }}$, where $s=1 \mathrm{~g} \mathrm{~cm}^{-3}, M=18, m_{\mathbf{H}}=$ hydrogen mass. This gives $\sigma_{\mathrm{H}_{2} \mathrm{O}}=0.32 \times 10^{-18} \mathrm{~cm}^{2}$ and $n_{\mathrm{H}_{2} \mathrm{O}} / n_{\mathrm{CO}} \simeq 5$. This is consistent with the values obtained for a wide variety of cloud conditions (d'Hendecourt et al., 1985). In HL Tau, as expected from the evidence for heating of the icy mantle (van de Bult et al., 1985), the solid $\mathrm{CO}$ is substantially reduced, being $\tau_{\mathrm{CO}}<0.05$, leading to $n_{\mathrm{H}_{2} \mathrm{O}} / n_{\mathrm{CO}}>20$.

\section{Cyclic Evolution of Grains}

The evolutionary picture of dust which is emerging is a cyclic one in which the particles find themselves alternately in diffuse clouds and in molecular clouds. A small silicate core captured within a molecular cloud gradually builds up an inner mantle of organic 
refractory material which has been produced by photoprocessing of the volatile ices. Within the dense clouds critical densities lead to star formation and subsequent ejection of some of the cloud material back into the surrounding space. Much of this material finding itself in a very tenuous low-density environment expands to the diffuse cloud phase. Dust particles in the diffuse medium are subjected to numerous destructive processes which rapidly erode the outer volatile mantles away and, in fact, erode part of the organic refractory material. It is important to note that without their organic refractory mantles the silicate cores could not survive. The rate of destruction of pure silicate grain leads to a maximum lifetime of $\tau_{\mathrm{Sil}} \simeq 4 \times 10^{8} \mathrm{yr}$ (Draine and Salpeter, 1979) which converts to a mass loss rate of $\mathrm{d} \rho_{\mathrm{Sil}} / \mathrm{d} t=-5 \times 10^{-43} \mathrm{~g} \mathrm{~cm}^{-3} \mathrm{~s}^{-1}$. Assuming a mass loss rate from M-stars of $1 M_{\odot} \mathrm{yr}^{-1}$ and a full cosmic abundance silicate production leads to a production rate for silicates of $\mathrm{d} \rho_{\mathrm{Sil}} / \mathrm{d} t<10^{-45} \mathrm{~g} \mathrm{~cm}^{-3} \mathrm{~s}^{-1}$ which is 100 times lower than the destruction rate. On the other hand, the production rate of the O.R. of $\mathrm{d} \rho_{\text {O.R. }} / \mathrm{d} t \simeq 10^{-41} \mathrm{~g} \mathrm{~cm}^{-3} \mathrm{~s}^{-1}$ is adequate to replenish the mantle material lost in the diffuse cloud phase even if the O.R. is somewhat less tough than the silicates. Therefore, silicate core-organic refractory mantle grains survive the diffuse cloud phase to reenter the molecular cloud phase.

The mean star production rate of $1-2 M_{\odot} \mathrm{yr}^{-1}$ implies an interstellar medium turnover time of $\sim 5 \times 10^{9} \mathrm{yr}$ so that this is the absolute maximum lifetime of a dust particle no matter how resistant to destruction. If we use a mean molecular cloud-diffuse cloud period of $2 \times 10^{8} \mathrm{yr}\left(10^{8} \mathrm{yr}\right.$ in each) then a typical grain anywhere in space will have undergone at least 20 cycles so that, for example, the typical diffuse cloud dust particle age is $\gtrsim 10^{9} \mathrm{yr}$ and consists of a mix of particles which have undergone a wide variety of photoprocessing. Note that the organic refractory mantles are subjected to the highest photoprocessing rates in the diffuse cloud phase. This would imply that the organic refractory mantle on a grain is not a homogeneous substance. A result of sequential formation (in the molecular cloud phase) and of intense photoprocessing (in the diffuse cloud phase) would lead to a layering in which the innermost layers have been the most irradiated and the outermost layer is first generation organic refractory.
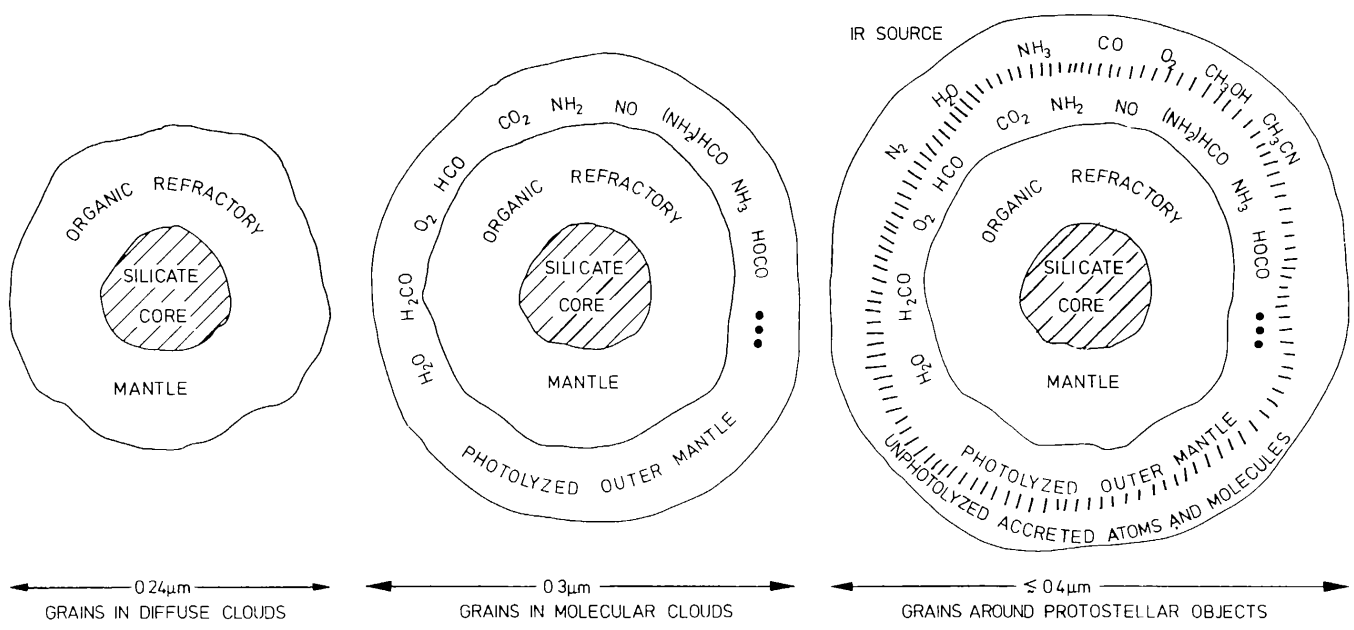

Fig. 8. Cross-section of grains at various stages of evolution. 
Because of this kind of layering, and the fact that the grains are of vairous ages leads one to expect average homogeneity of diffuse cloud grains both in size and structure which is observed as a uniformity in the visual extinction curve and a rather structureless $3.4 \mu \mathrm{m}$ feature. In other words, diffuse cloud grains represent a steady-state average of grains of a multiplicity of chemical and physical histories.

A schematic representation of grains in the various regions of space is shown in Figure 8. In the final stage of cloud condensation we may expect that all remaining (condensable) molecules will have accreted onto the dust. In addition, the very small $(\lesssim 0.01 \mu \mathrm{m})$ particles will be collected and trapped within the outer volatile mantle.

\section{References}

Aanestad, P. A. and Greenberg, J. M.: 1983, Astrophys. J. 272, 551.

Butchart, I., McFadzean, A. D., Whittet, D. C. B., Geballe, T. R., and Greenberg, J. M.: 1986, Astron. Astrophys. 154, L5.

Cohen, M.: 1983, Astrophys. J. 270, L69.

d'Hendecourt, L. B., Allamandola, L. J., Baas, F., and Greenberg, J. M.: 1982, Astron. Astrophys. 109, L12. d'Hendecourt, L. B., Allamandola, L. J., and Greenberg, J. M.: 1985, Astron. Astrophys. 152, 130.

d'Hendecourt, L. B., Allamandola, L. J., Grim, R. A., and Greenberg, J. M.: 1986, Astron. Astrophys. 158, 119.

Draine, B. T. and Salpeter, E. E.: 1979, Astrophys. J. 231, 438.

Greenberg, J. M.: 1973, in M. A. Gordon and L. E. Snyder (eds.), Molecules in the Galactic Environment, Wiley, New York, p. 94.

Greenberg, J. M.: 1979, in B. E. Westerlund (ed.), Stars and Star Systems, D. Reidel Publ. Co., Dordrecht, Holland, p. 173.

Greenberg, J. M.: 1982a, in J. E. Beckman and J. P. Phillips (eds.), Submillimetre Wave Astronomy, Cambridge Univ. Press, Cambridge, p. 261.

Greenberg, J. M.: 1982b, in L. L. Wilkening (ed.), Comets, Univ. of Arizona Press, Tucson, p. 131.

Greenberg, J. M.: 1986, in F. Israel (ed.), Proceedings of IRAS Symposium 'Light on Dark Matter', D. Reidel Publ. Co., Dordrecht, Holland.

Greenberg, J. M. and Hong, S. S.: 1975, in G. B. Field and A. G. W. Cameron (eds.), The Dusty Universe, Neal Watson Academic Publ., p. 31.

Greenberg, J. M., van de Bult, C. E. P. M., and Allamandola, L. J.: 1983, J. Phys. Chem. 87, 4243.

Greenberg, J. M. Yencha, A. J., Corbett, J. W., and Frisch, H. L.: 1972, Mém. Soc. Roy. Soc. Liège 3, 425.

Lacy, J. H., Baas, F., Allamandola, L. J., Persson, S. E., McGregor, P. J., Lonsdale, C. J., Geballe, T. R., and van de Bult, C. E. P. M.: 1984, Astrophys. J. 276, 533.

Léger, A., Jura, M., and Omont, A.: 1985, Astron. Astrophys. 144, 147.

Mann, A. P. C. and Williams, D. A.: 1980, Nature 283, 721.

Mathis, J. S., Rumpl, W., and Nordsieck, K. H.: 1979, Astrophys. J. 217, 425.

Moore, M. H. and Donn, B.: 1982, Astrophys. J. 257, L47.

Schutte, W. and Greenberg, J. M.: 1986, in F. Israel (ed.), IRAS Symp. 'Light on Dark Matter', D. Reidel Publ. Co., Dordrecht, Holland.

Soifer, R. W., Puetter, R. C., Russell, R. W., Willner, S. P., Harvey, R. M., and Gillett, F. C.: 1979, Astrophys. J. 232, L53.

Strazzulla, G., Cataliotti, R. S., Calcagno, L., and Foti, G.: 1984, Astron. Astrophys. 133, 77.

Van de Bult, C. E. P. M., Greenberg, J. M., and Whittet, D. C. B.: 1985, Monthly Notices Roy. Astron. Soc. 214, 289.

Van de Hulst, H. C.: 1949, Rech. Astron. Utrecht 11, part 2.

Van IJzendoorn, L. J.: 1985, Ph.D. Thesis, Univ. of Leiden, Holland.

Whittet, D. C. B., Bode, M. F., Longmore, A. J., Baines, D. W. T., and Evans, A.: 1983, Nature 303, 298.

Whittet, D. C. B., Longmore, A. J., and McFadzean, A. D.: 1985, Monthly Notices Roy. Astron. Soc. 216, $45 \mathrm{P}$.

Willner, S. P., Russell, R. W., Puetter, R. C., Soifer, B. T., and Harvey, P. N.: 1979, Astrophys. J. 229 , L65. 


\section{Discussion}

P. G. Mezger: Would these small grains without the silicate core be the same material which in earlier talks we referred to as yellow stuff?

J. M. Greenberg: That is right in part. At least those which give the $220 \mathrm{~nm}$ hump, although they require further photoprocessing.

$P$. G. Mezger: For me the thing would be more convincing if you could produce an extinction curve as far as $1000 \mu \mathrm{m}$.

J. M. Greenberg: We met some difficult problems. I am not sure that the PAHs are important enough, I agree with you they probably are not. What I do believe is that small particles may actually be responsible for the near-infrared extinction. That is we also have carbon particles in our distribution and in our grain models we have used graphite because we do not have anything better yet. No matter which carbon you use problems of getting the near-infrared extinction remain.

But the question is: Do these $220 \mathrm{~nm}$ particles produce that near-infrared extinction? The answer to that is that I do not know yet. I know about the specific absorptive properties $(3.4,3.0 \mu \mathrm{m})$, but I do not know what the overall absorption properties are, let us say at 4 or $10 \mu \mathrm{m}$.

I believe that there is carbon in space, however I found very great difficulties with graphite for a number of reasons. We see sufficient evidence for smoky stars to believe that carbon is coming out from stars. The question is whether it is graphite. At a meeting in Les Houches two weeks ago nobody really believed in graphite. They were talking about hydrogenated amorphous carbon.

To summarize the situation: We do not know what is producing the variability in the NIR. I think the observed differences are real.

E. Krügel: There is a very interesting point about the accretion on the grains which you mentioned. In a dense cloud the accretion is so effective that all the molecules should have gone and you solved the riddle in that you say that we have some mechanism by which they evaporate from the grains. You say that you obtain the kinetic energies for evaporation from grain-grain collisions with velocities of the order of $0.1 \mathrm{~km} \mathrm{~s}^{-1}$. I assume this is for pure inelastic collisions. But there are some cloud cores where molecules are observed with very narrow lines and of course the grains cannot have thermal velocities, they can only have turbulent velocities. But in these very dense cold cores there is very little turbulence. I have the suspicion that you run into trouble not only from the energetic point of view but if the velocities are too low you do not get enough collisions to get the balance which is observed.

J. M. Greenberg: First of all I do not know hold old the cores are. If you were talking about $10^{6} \mathrm{yr}$ there is no problem, you would still see them. In terms of the turbulence 
there is one way to answer. If we do not observe turbulence the mechanism of grain-grain collisions cannot work. The question then remains: Is there another possible triggering mechanism? Jura and Léger proposed the possibility of explosions following the passage of cosmic iron particles. They calculated the flux on the grains in a galactic cloud and apparently the cross sections are comparable to those of grain-grain collisions.

Th. Henning: Is there any observation of the absorption from mantles at the longwavelength wing of the silicate band?

$J . M$. Greenberg: Yes, I believe so. There is some evidence that there is in some regions an existence of an extra ice absorption.

H.J. Habing: From IRAS data we have nothing conclusive yet. We have some problems with the determination of a 'standard' continuum. 